\title{
The magnetic field in the X-ray binary Cyg X-1
}

\author{
Eugenia A. Karitskaya ${ }^{1}$, N. G. Bochkarev ${ }^{2}$, S. Hubrig ${ }^{3}$, \\ Yu. N. Gnedin ${ }^{4}$ M. A. Pogodin ${ }^{4}$, R. V. Yudin ${ }^{4}$, \\ M. I. Agafonov ${ }^{5}$ and O. I. Sharova ${ }^{5}$ \\ ${ }^{1}$ Astronomical Institute of RAS, 48 Pyatnitskaya str., Moscow 119017, Russia, \\ email: karitsk@sai.msu.ru \\ ${ }^{2}$ Sternberg Astronomical Institute 13 Universitetskij pr., Moscow 119991, Russia \\ ${ }^{3}$ ESO, Santiago, Chile \\ ${ }^{4}$ Pulkovo Observatory RAS, St. Petersburg 196140, Russia \\ ${ }^{5}$ Radiophysical Research Institute, Nizhny Novgorod 603950, Russia
}

\begin{abstract}
VLT FORS 1 observations indicate the presence of a variable significant magnetic field in the X-ray binary Cyg X-1. The importance of this investigation comes from the fact that it rules one of the most significant $\mathrm{BH}$ manifestations: the X-ray millisecond flickering, usually related to reconnection of magnetic lines in the innermost part of the accretion disc.
\end{abstract}

Keywords. X-ray binaries - black hole physics - magnetic fields - spectropolarimetry - accretion

\section{Introduction}

The first prediction of the presence of a magnetic field in Cyg X-1 was done by V. F. Shvartsman (1971). Many previous attempts to measure the magnetic field have been unsuccessful providing only the upper limit for the strength of the magnetic field (e.g. Gnedin et al. 2003).

\section{Observations and analysis}

Between June 18 and July 9, 2007 we obtained in service mode six FORS 1 spectropolarimetric spectra. We used the GRISM $1200 \mathrm{~B}$ and a 0.4 arcsec slit to obtain a spectral resolution of 4000. This grism covers the spectral range $3680-5129 \AA$ which includes Balmer lines from $\mathrm{H} \beta$ to $\mathrm{H} \epsilon$. At that time Cyg X-1 was in X-ray hard state. For each observation we usually took a number of continuous series of two exposures. More details on the observing technique with FORS 1 and data reduction can be found elsewhere (Hubrig et al. 2004 and references therein). Using the method described in the same place we obtained the mean longitudinal magnetic field $\left\langle B_{z}\right\rangle$ averaged over the stellar hemisphere visible at the time of observation of the component of the magnetic field parallel to the line of sight, weighted by the local emergent spectral line intensity.

As an important step, before the assessment of $\left\langle B_{z}\right\rangle$, we removed all the spectral features not belonging to the photosphere of the Cyg X-1 optical component (O9.7 Iab): telluric and interstellar lines, CCD defects, the He II $\lambda 4686 \AA$ emission line, lines with strong P Cyg components. The measurements of the longitudinal magnetic field at different orbital phases are presented in Fig. 1. It is quite possible that near the orbital 


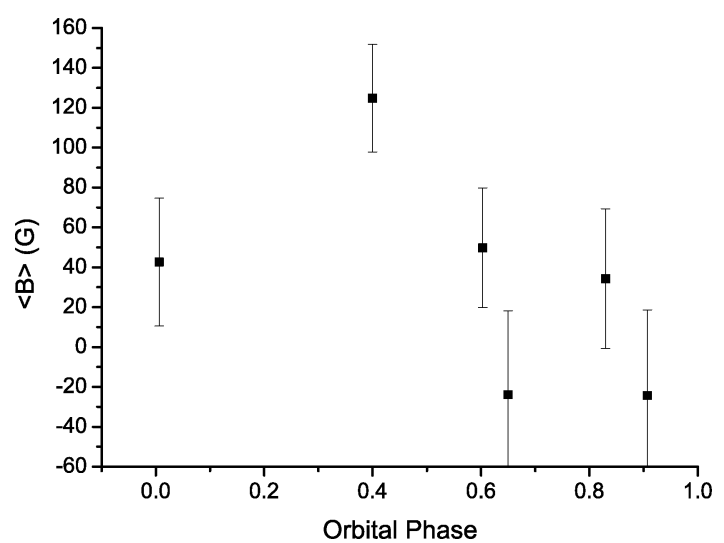

Figure 1. The longitudinal magnetic field plotted over the orbital phase $(\mathrm{P}=5.6$ days $)$ of Cyg X-1.

phase 0.5 (having the X-ray source in front) we are observing the optical component in the vicinity of the magnetic pole with the $\left\langle B_{z}\right\rangle$ value close to $130 \mathrm{G}$ on $5 \sigma$ level.

On the second step of our research we investigated $H e I I \lambda 4686 \AA$ spectral line separately. The accuracy of magnetic field measuring over one line lower than from all lines. Estimation on 2 sigma level was found only for orbital phase $0.65\langle B z\rangle=-607 \pm 310 \mathrm{G}$. $\langle B z\rangle$ reality is confirmed: by Zeeman S-wave in $\mathrm{V}$-spectrum and by its correspondence to the $d I(\lambda) / d \lambda$ wave.

\section{Discussion}

The Doppler tomogram of the system based on the HeII $\lambda 4686$ Åline using FORS 1 Stokes I spectra shows that the line emission component originates in the outer regions of the accretion structure. We used the Doppler tomogram building technique of Agafonov, (2004), so-called radio astronomical approach. The magnetic field strength of $600 \mathrm{G}$ is an expected value since the gas stream from the star carrying the magnetic field onto the accretion structure is compressed by interaction with its outer rim by a factor of $6-10$ at a distance from the black hole $\mathrm{D}=6 * 10^{11} \mathrm{~cm}$. According to the standard model of the disc accretion, such a result would correspond to the near-Black-Hole magnetic field value of $\sim 10^{8}-10^{9} \mathrm{G}$ and thus the value of its energy would be sufficient to explain Cyg X-1 X-ray millisecond flickering.

\section{Acknowledgements}

We would like to acknowledge ESO for grants for VLT observations in service mode. The work was supported by RFBR by grants 06-02-16234, 06-02-16843, 07-02-00535 and Sci.Schole 6110.2008.2.

\section{References}

Agafonov, M. I. 2004, AN 325, 259

Gnedin, Yu. N. et al. 2003, astro-ph/4158G

Hubrig, S., Kurtz, D. W., Bagnulo, S. et al. 2004, A\&A 415, 661

Shvartsman, V. F. 1971, AZh 48, 479 